\title{
Chapter 4 \\ Oral Microbiota in Crevices Around Dental \\ Implants: Profiling of Oral Biofilm
}

\author{
Takuichi Sato, Yoshiaki Kawamura, Keiko Yamaki, Naoko Ishida, \\ Lingyang Tian, Yasuhisa Takeuchi, Kazuhiro Hashimoto, Yuki Abiko, \\ Gen Mayanagi, Jumpei Washio, Junko Matsuyama, and \\ Nobuhiro Takahashi
}

\begin{abstract}
Large numbers of bacteria $\left(>10^{6} / \mathrm{mm}^{2}\right)$ generally inhabit the surface of the oral cavity, particularly at the interface between teeth and gingiva, as an oral biofilm (microbiota). The establishment of anaerobic bacterial culture and molecular biological techniques has enabled us to isolate and detect various bacterial species from oral biofilm. It has been estimated that more than 600 bacterial species
\end{abstract}

T. Sato $(\varangle) \cdot$ L. Tian • Y. Abiko • J. Washio • N. Takahashi

Division of Oral Ecology and Biochemistry, Tohoku University Graduate School of Dentistry, Sendai 980-8575, Japan

e-mail: tak@m.tohoku.ac.jp

Y. Kawamura

Department of Microbiology, Aichigakuin University School of Pharmacy, Nagoya 464-8650, Japan

K. Yamaki • K. Hashimoto

Division of Periodontology and Endodontology, Tohoku University Graduate School of

Dentistry, Sendai 980-8575, Japan

N. Ishida

Division of Oral Ecology and Biochemistry, Tohoku University Graduate School of Dentistry, Sendai 980-8575, Japan

Division of Advanced Prosthetic Dentistry, Tohoku University Graduate School of Dentistry, Sendai 980-8575, Japan

Y. Takeuchi

Division of Advanced Prosthetic Dentistry, Tohoku University Graduate School of Dentistry, Sendai 980-8575, Japan

G. Mayanagi

Division of Oral Ecology and Biochemistry, Tohoku University Graduate School of Dentistry, Sendai 980-8575, Japan

Research Unit for Interface Oral Health Science, Tohoku University Graduate School of Dentistry, Sendai 980-8575, Japan

J. Matsuyama

Division of Pediatric Dentistry, Niigata University Graduate School of Medical and Dental

Sciences, Niigata 951-8514, Japan 
inhabit the oral cavity. Nevertheless, the oral cavity is considered healthy when the oral microbiota is composed of indigenous bacteria. Numerous environmental changes in the oral cavity may lead to accumulation of dental caries-associated or periodontitis-associated bacteria, resulting in the initiation of dental caries or periodontitis, respectively. The environment in crevices around dental implants is considered similar to that in subgingival sulcus, such as neutral $\mathrm{pH}$, anaerobiosis and rich nutrition (e.g., amino acids and peptides). The environment may be supportive of anaerobic growth of the bacteria in microbiota in crevices around implants, particularly at the interface between histocompatible artificial material and mucosal epithelium. The microbiota may trigger inflammation in the tissue around the implants. In this article, the current topics on the profiling of oral microbiota in crevices around implants are reviewed.

Keywords Bacteria $\bullet$ Dental implant $\bullet$ Oral microbiota $\bullet$ Profiling

\subsection{Introduction}

Large numbers of bacteria $\left(>10^{6} / \mathrm{mm}^{2}\right)$ generally inhabit the surface of the oral cavity, particularly at the interface between teeth and gingiva, as an oral biofilm (microbiota). The establishment of anaerobic bacterial culture and molecular biological techniques has enabled us to isolate and detect various bacterial species from oral biofilm. Currently, it has been estimated that more than 600 bacterial species inhabit the oral cavity. Nevertheless, the oral cavity is considered healthy when the oral microbiota is composed of indigenous bacteria.

\subsubsection{Quantitative and Qualitative Analyses of Oral Biofilm}

For the past several decades, with the development of techniques for culturing obligate anaerobes, in particular, adoption of the well-maintained anaerobic glove box system permitted the efficient recovery of obligate anaerobes from oral cavities and lesions. Importantly, all plates, media, buffer solutions and experimental instruments are kept in an anaerobic glove box for at least $24 \mathrm{~h}$ before use. To ensure strictly anaerobic conditions in the glove box, reduction of methyl viologen $(-446 \mathrm{mV})$ is carefully monitored whenever experimental procedures are carried out. By adopting these exacting anaerobic techniques, the microbiota of oral biofilm has been shown to consist mainly of obligate anaerobes [1-5].

At present, the identification of obligate anaerobes is generally performed utilizing molecular biological techniques [6-12]. For instance, the bacterial 16S ribosomal RNA gene sequences are amplified by PCR, and partial sequences are then compared with those from the GenBank database using the BLAST search program through the National Center for Biotechnology Information website. Bacterial species are determined by percent sequence similarity ( $>97 \%)$. 


\subsubsection{Oral Ecology: Environmental Factors Affecting Oral Biofilm}

Numerous environmental changes in the oral cavity may lead to an accumulation of dental caries-associated or periodontitis-associated bacteria, resulting in the initiation of dental caries or periodontitis, respectively. The environment in crevices around dental implants is considered similar to that in subgingival sulcus, such as neutral $\mathrm{pH}$, anaerobiosis and rich nutrition (e.g., amino acids and peptides). The environment may be supportive of anaerobic growth in the crevices around implants, particularly at the interface between histocompatible artificial material and mucosal epithelium. The microbiota may trigger inflammation in the tissue around the implants.

In this article, the current topics related to profiling of oral microbiota in crevices around implants are reviewed.

\subsection{Nutritional and Environmental Aspects of Dental Implants}

Nutrition for bacteria are supplied by the fluids around implants (PICF; periimplant crevicular fluids) and teeth (GCF; gingival crevicular fluids), thus, fluid volume and contents, as well as $\mathrm{pH}$, are considered to be critical to the health of dental implants.

\subsubsection{Fluid Volume}

Fluid volume with healthy implants was $2.17 \pm 2.09 \mu \mathrm{L}$ (range of Periotron 22.635.2 units) and that with healthy teeth was $3.49 \pm 2.26 \mu \mathrm{L}$ (36.3-56.6 units) ( $\mathrm{n}=7$, mean age, 54.2 years) [13]. Similarly, Apse et al. [14] reported that fluid volumes were Periotron $57.1 \pm 37.5$ units and $54.8 \pm 28.9$ units, for healthy implants $(n=28)$ and healthy teeth $(n=19)$, respectively. There were no significant differences between healthy implants and healthy teeth, suggesting similar conditions between healthy implants and healthy teeth.

\subsubsection{Fluid Constituents}

In terms of profile of crevicular fluid constituents such as inflammatory markers in healthy PICF and GCF are reportedly similar, e.g., $\alpha 2$-macroglobulin $(17$ and $16 \mathrm{ng} / \mu$ g albumin), $\alpha 1$-antitrypsin (33 and 48), transferrin (34 and 47), lactoferrin (17 and 40) 
and immunoglobulin $\mathrm{G}$ against Porphyromonas gingivalis (82 and 102), respectively [15]. These trends were also observed in inflamed status (peri-implantitis and periodontitis), e.g., $\alpha 2$-macroglobulin (20 and $15 \mathrm{ng} / \mu \mathrm{g}$ albumin), $\alpha 1$-antitrypsin (42 and 46), transferrin (35 and 31), lactoferrin (14 and 24) and immunoglobulin $\mathrm{G}$ against Porphyromonas gingivalis (47 and 43), respectively [15].

\subsubsection{Environmental Condition: Fluid pH}

Fluid $\mathrm{pH}$ of healthy PICF was 6.82 (range 6.30-7.70) and that of healthy GCF was 6.90 (range $6.50-8.50$ ). On the other hand, the ranges of fluid $\mathrm{pH}$ for periimplantitis and periodontitis were 5.63-8.50 and 7.20-7.70, respectively [16].

\subsection{Microbiota Around Implants}

\subsubsection{Quantitative and Qualitative Analysis of Microbiota in PICF}

Bacterial counts in healthy PICF $(n=10)$ were $(0.8 \pm 2.0) \times 10^{6}[17]$, while those in healthy GCF $(\mathrm{n}=7)$ were $(7.6 \pm 8.6) \times 10^{8}$ [7]. In inflamed status, $(7.5 \pm 9.8) \times$ $10^{7}$ bacteria were recovered from plate crevices $(n=3)$ for orthodontic treatment [7], while $(5.8 \pm 2.8) \times 10^{6}$ and $(1.6 \pm 1.5) \times 10^{7}$ bacteria were found in periodontal pockets ( $n=7$ and $n=5$ from [4] and [8], respectively).

The proportion of obligate anaerobes among the microbiota of healthy PICF was $21 \%$ [17], and that of healthy GCF was $31 \%$ [7]. With regard to bacterial composition, Actinomyces (17\%), Campylobacter (12\%), Fusobacterium (10\%), Selenomonas (10\%), Streptococcus (8.2\%), Lepotrichia (7.6\%), Prevotella (7.1\%), Neisseria (6.5\%), Veillonella (6\%), Dialister (3.3\%) and Haemophilus $(2.7 \%)$ were predominant in healthy plate crevices, while Actinomyces $(37 \%)$, Streptococcus (20\%), Veillonella $(7.5 \%)$, Olsenella $(6.2 \%)$, Prevotella $(4.8 \%)$, Fusobacterium (2.7\%), Parvimonas (2.7\%), Selenomonas (2.1\%), Neisseria (2.1\%), Capnocytophaga (2.1\%), Gemella (2.1\%), Rothia (2.1\%) and Haemophilus $(1.4 \%)$ were predominant in healthy GCF [7]. Under the viewpoint that major anaerobes were Campylobacter, Fusobacterium, Selenomonas, Prevotella, Veillonella and Dialister, and major facultative anaerobes were Actinomyces, Streptococcus, Neisseria and Haemophilus, a similarity were suggested between healthy plate crevices and healthy GCF.

This suggests that the microbiota around implants is similar to that of gingival sulcus with regard to bacterial density and proportion of anaerobes in microbiota. Therefore, similarly to the maintenance of the teeth with periodontal pockets, treatments with dental implants require strict self-oral care and regular professional plaque control in order to prevent infection. 


\subsubsection{Metagenome (Pyrosequencing) Analysis of Microbiota in PICF}

In contrast, inflammatory and immune responses of peri-implant mucosa to microbiota around implants have not been reported in detail. Thus, it is possible that particular microbiota may be formed around dental implants. Indeed, recent pyrosequencing analyses have shown that microbiota in healthy PICF was distinct from that in healthy GCF, and that the currently accepted theory on the transmission from the tooth to the implant surface requires reexamination $[18,19]$. More specifically, utilizing the pyrosequencing technique, Kumar et al. [18] reported that Prevotella, Treponema, Leptotrichia, Streptococcus mutans, Butyrivibrio and Lactococcus were significantly present in healthy PICF, while non-mutans streptococci, Fusobacterium, Actinomyces, Granulicatella, Dialister, Veillonella, Neisseria, Corynebacterium, Synergistes and Arthrobacter were significantly present in healthy GCF. In addition, Dabdoub et al. [19] reported that Actinomyces gerencseriae, Actinomyces bovis, Veillonella dispar, Haemophilus influenza, Streptococcus minor, Mycoplasma faucium, Streptococcus macedonicus, Streptococcus pseudoporcinus, Unclassified Bacillales, Actinomyces radicidentis, Streptococcus infantis, Actinomyces meyeri, Streptococcus ursoris and Veillonella spp. were significantly present in healthy PICF, while Caulobacter spp., Peptostreptococcus anaerobius, Unclassified Rs-045, Desulfobulbus spp. and Bulleidia spp. were significantly present in healthy GCF.

With the improvement of materials and techniques of dental implants, the environment around dental implants will possibly change markedly, and thus a comprehensive analysis of microbiota, as well as the development of novel markers in the environment, is required in order to elucidate the etiological role in periimplant diseases.

Acknowledgements This study was supported in part by Grants-in-Aid for Scientific Research ( 25462945 to KY, 25463237 to JM, 25861785 to KH, 24390511, 25670777, 26462869 to TS) from the Japan Society for the Promotion of Science.

Open Access This chapter is distributed under the terms of the Creative Commons Attribution Noncommercial License, which permits any noncommercial use, distribution, and reproduction in any medium, provided the original author(s) and source are credited.

\section{References}

1. Hoshino E. Predominant obligate anaerobes in human carious dentin. J Dent Res. 1985;64:1195-8.

2. Hoshino E, Sato M, Sasano T, Kota K. Characterization of bacterial deposits formed in vivo on hydrogen-ion-sensitive field-effect transistor electrodes and enamel surfaces. Jpn J Oral Biol. 1989;31:102-6.

3. Ando N, Hoshino E. Predominant obligate anaerobes invading the deep layers of root canal dentin. Int Endod J. 1990;23:20-7. 
4. Uematsu H, Hoshino E. Predominant obligate anaerobes in human periodontal pockets. J Periodontal Res. 1992;27:15-9.

5. Sato T, Hoshino E, Uematsu H, Noda T. Predominant obligate anaerobes in necrotic pulps of human deciduous teeth. Microb Ecol Health Dis. 1993;6:269-75.

6. Washio J, Sato T, Koseki T, Takahashi N. Hydrogen sulfide-producing bacteria in tongue coating and their relationship with oral malodour. J Med Microbiol. 2005;54:889-95.

7. Sato R, Sato T, Takahashi I, Sugawara J, Takahashi N. Profiling of bacterial flora in crevices around titanium orthodontic anchor plates. Clin Oral Implants Res. 2007;18:21-6.

8. Hashimoto K, Sato T, Shimauchi H, Takahashi N. Profiling of dental plaque microflora on root caries lesions and the protein-denaturing activity of these bacteria. Am J Dent. 2011;24:295-9.

9. Komori R, Sato T, Takano-Yamamoto T, Takahashi N. Microbial composition of dental plaque microflora on first molars with orthodontic bands and brackets, and the acidogenic potential of these bacteria. J Oral Biosci. 2012;54:107-12.

10. Sato T, Yamaki K, Ishida N, Hashimoto K, Takeuchi Y, Shoji M, Sato E, Matsuyama J, Shimauchi H, Takahashi N. Cultivable anaerobic microbiota of infected root canals. Int J Dent. 2012;2012:609689.

11. Takeuchi Y, Nakajo K, Sato T, Koyama S, Sasaki K, Takahashi N. Quantification and identification of bacteria in acrylic resin dentures and dento-maxillary obturator-prostheses. Am J Dent. 2012;25:171-5.

12. Hasegawa A, Sato T, Hoshikawa Y, Ishida N, Tanda N, Kawamura Y, Kondo T, Takahashi N. Detection and identification of oral anaerobes from intraoperative bronchial fluids of patients with pulmonary carcinoma. Microbiol Immunol. 2014;58:375-81.

13. Murata M, Tatsumi J, Kato Y, Suda S, Nunokawa Y, Kobayashi Y, Takeda H, Araki H, Shin K, Okuda K, Miyata T, Yoshie H. Osteocalcin, deoxypyridinoline and interleukin-1beta in periimplant crevicular fluid of patients with peri-implantitis. Clin Oral Implants Res. 2002;13:637-43.

14. Apse P, Ellen RP, Overall CM, Zarb GA. Microbiota and crevicular fluid collagenase activity in the osseointegrated dental implant sulcus: a comparison of sites in edentulous and partially edentulous patients. J Periodontal Res. 1989;24:96-105.

15. Adonogianaki E, Mooney J, Wennström JL, Lekholm U, Kinane DF. Acute-phase proteins and immunoglobulin G against Porphyromonas gingivalis in peri-implant crevicular fluid: a comparison with gingival crevicular fluid. Clin Oral Implants Res. 1995;6:14-23.

16. Nyako EA, Watson CJ, Preston AJ. Determination of the $\mathrm{pH}$ of peri-implant crevicular fluid in successful and failing dental implant sites: a pilot study. Arch Oral Biol. 2005;50:1055-9.

17. Mombelli A, van Oosten MA, Schurch Jr E, Land NP. The microbiota associated with successful or failing osseointegrated titanium implants. Oral Microbiol Immunol. 1987;2:145-51.

18. Kumar PS, Mason MR, Brooker MR, O'Brien K. Pyrosequencing reveals unique microbial signatures associated with healthy and failing dental implants. J Clin Periodontol. 2012;39:425-33.

19. Dabdoub SM, Tsigarida AA, Kumar PS. Patient-specific analysis of periodontal and periimplant microbiomes. J Dent Res. 2013;92(12 Suppl):168S-75. 